Article

\title{
Kinetics of the Aqueous-Phase Copolymerization of MAA and PEGMA Macromonomer: Influence of Monomer Concentration and Side Chain Length of PEGMA
}

\author{
Iñaki Emaldi ${ }^{1}$, Shaghayegh Hamzehlou ${ }^{1}$, Jorge Sanchez-Dolado ${ }^{2}$ and Jose R. Leiza ${ }^{1, *}$ \\ 1 POLYMAT and Kimika Aplikatua Saila, Kimika Zientzien Fakultatea, University of the Basque Country \\ UPV/EHU, Joxe Mari Korta Zentroa, Tolosa Hiribidea 72, 20018 Donostia-San Sebastián, Spain; \\ inaki.emaldi@ehu.eus (I.E.); shaghayegh.hamzehlou@ehu.eus (S.H.) \\ 2 Sustainable Construction Division, Tecnalia Research \& Innovation, Parque Tecnológico de Bizkaia, c/Geldo, \\ Edificio 700, 48160 Derio, Spain; jorge.dolado@tecnalia.com \\ * Correspondence: jrleiza@ehu.eus; Tel.: +34-943-01-5329
}

Academic Editor: Alexander Penlidis

Received: 15 March 2017; Accepted: 14 April 2017; Published: 20 April 2017

\begin{abstract}
An in situ nuclear magnetic resonance spectroscopy (NMR) technique is used to monitor the aqueous-phase copolymerization kinetics of methacrylic acid (MAA) and poly(ethylene glycol) methyl ether methacrylate (PEGMA) macromonomers. In particular, the study analyses the effect of the number of ethylene glycol (EG) groups along the lateral chains of PEGMA and is carried out under fully ionized conditions of MAA at different initial monomer ratios and initial overall monomer concentrations (5-20 wt \% in aqueous solution). The composition drift with conversion indicates that PEGMA macromonomer is more reactive than MAA. Individual monomer consumption rates show that the rates of consumption of both monomers are not first order with respect to overall concentration of the monomer. The reactivity ratios estimated from the copolymerization kinetics reveal, that for the short PEGMA, the reactivity ratios $r_{M A A}$ and $r_{P E G M A}$ increase with the solids content (SC). A totally different trend is obtained for the longer PEGMA, whose reactivity ratio $\left(r_{P E G M A 23}\right)$ decreases with solids content, whereas the reactivity ratio of MAA remains roughly constant.
\end{abstract}

Keywords: aqueous-phase copolymerization; polyethylene glycol methacrylate monomers; reactivity ratios; ionization degree; solids content

\section{Introduction}

During the last few years, the use of monomers with a polyethylene glycol (PEG) side chain in the synthesis of polymeric materials (as comonomers in aqueous-phase solution copolymerization [1-3] or as reactive stabilizers in several heterogeneous polymerization systems) [4-7] has gained increasing attention. Water-soluble polymers are used in a variety of applications including coatings, cosmetics, antiflocculants, textiles, superabsorbers and water treatment [8-10]. These materials are generally produced via free-radical (co)polymerization in aqueous solution $[9,11,12]$. Although of great industrial importance, understanding of the aqueous polymerization kinetics is far from reaching the understanding achieved in organic solvents. One major difference found between aqueous and organic solvents is that the propagation rate constant of water-soluble monomers, in addition to temperature, depends on other variables such as monomer concentration, $\mathrm{pH}$ and ionic strength of the aqueous medium, which makes the kinetics substantially more complex than in organic systems, especially when dealing with copolymerization processes. 
Thus, in the last twenty years, several research groups have systematically analysed the effect of monomer concentration on the propagation rate coefficient, $k_{p}$, of the most common water-soluble monomers including acrylic acid (AA) [13-16], acrylamide (AM) [17,18], methacrylic acid (MAA) [19-21], $N$-vinyl pyrrolidone (NVP) [22] and $N$-vinyl formamide (NVF) [23,24]. Very recently, Smolne et al. [1] have also determined the propagation rate coefficient of poly(ethylene glycol) methyl ether methacrylate (PEGMA) in aqueous phase at different concentrations.

All these studies shared a common finding: the $k_{p}$ of these monomers decrease with increasing the concentration of monomer. This decrease is attributed to a reduction in the Arrhenius pre-exponential factor, which is related to entropic factors (independent of temperature) due to the influence of competitive hydrogen bonding between the transition-state structure and side groups of the monomer and water. The reduction can be up to one order of magnitude going from bulk to very dilute conditions $[1,19,21]$. In addition to the effect of the monomer concentration on the $k_{p}$, the ionization degree also affects the kinetics of acidic water-soluble monomers like AA and MAA. For instance, for MAA, one of the monomers used in this work, Lacík et al. [19-21,25] have determined the effect of the ionization degree (from non-ionized to fully ionized conditions) and found that the $k_{p}$ significantly reduces on going from non-ionized form to the fully ionized form, and that this decrease diminishes as MAA concentration increases.

For PEGMA monomers, there are no reports on the effect of the $\mathrm{pH}$ of aqueous media on the propagation rate coefficient. However, it has been found that for AM [18] and NVP [22], the pH does not affect the propagation rate coefficient.

Copolymerization is generally implemented in order to obtain specific properties which are not attainable by homopolymers. Water-soluble poly(MAA-co-PEGMA) copolymers present comb-like structure, where the size of the lateral chain can be tuned by the use of PEGMA with different numbers of ethylene oxide (EO) groups. This class of comb copolymers under alkali conditions presents an anionic backbone and an uncharged side chain and are effective dispersants and/or lubricants of inorganic particles. The backbone is considered to drive adsorption mainly through electrostatic interactions with surfaces, while the side chains are chosen to be non-absorbing and to induce steric hindrance among adsorbed layers [26,27]. These water-soluble copolymers have found a tremendous success in cementitious formulations where the comb copolymer dispersants are known as polycarboxylate ether (PCE) superplasticizers (SPs). They are mainly used to produce concrete of greater strength and durability by making it possible to reduce the water content without sacrificing rheological properties. Two synthetic routes can be used to produce these comb copolymers: one route is the partial esterification of polymethacrylic acid (PMAA) with methoxy polyethylene glycol (MPEG) of different lengths employing acid or base catalyst and vacuum to remove water $[28,29]$. This method provides highly uniform MPEG chains with a statistical distribution of the PEG side chains along a PMAA backbone. The second route is via free-radical copolymerization of MAA and PEGMA, which is a much common route of producing this type of PCEs [30-32].

The composition and sequence distribution of the comonomers in the chain is expected to have a tremendous impact on the performance of the comb copolymer chains in the cementitious formulations. Therefore, it is of paramount importance to control the composition of the chains during the polymerization reaction in order to produce homogeneous copolymers and hence be able to understand their adsorption behaviour when implemented in cementitious formulations as SPs.

The reactivity ratios of the comonomers (MAA and PEGMA) are the key parameters to understand the type of copolymer chains produced during the copolymerization and thus the necessary parameters to develop control strategies aimed at controlling the instantaneous composition along the reaction. Unfortunately, the information about the reactivity ratios of the MAA/PEGMA comonomer pair in aqueous solution available in the literature is scarce [33-35] and reports for conditions where the MAA is fully ionized (basic conditions) are not available. Smith and Klier [33] found that the reactivity ratios measured in deuterated water $\left(\mathrm{D}_{2} \mathrm{O}\right)$ for the non-ionized MAA were very close to 1 for both monomers $\left(r_{M A A}=1.03\right.$ and $\left.r_{P E G M A}=1.02\right)$; however, by changing $50 \%$ of $\mathrm{D}_{2} \mathrm{O}$ by ethanol $\left(\mathrm{D}_{2} \mathrm{O} /\right.$ Ethanol $=50 / 50$, a continuous phase used in dispersion polymerization), the reactivity ratios measured for the monomers 
increased $\left(r_{M A A}=2.0\right.$ and $\left.r_{P E G M A}=3.6\right)$. Obviously this has an impact on the distribution of the monomer sequence in the chain that is random in water and more "blocky" in the case of water/ethanol.

Krivorotova et al. [35] have recently analyzed the copolymerization of MAA and PEGMA macromonomers carried out by conventional and controlled (RAFT, reversible addition fragmentation chain transfer) free-radical polymerization (FRP) in the mixture $\mathrm{D}_{2} \mathrm{O}$ /dioxane initiated by azobisisobutyronitrile (AIBN). MAA was also non-ionized in the experimental conditions investigated in this work. Interestingly, the authors considered two PEGMA macromonomers differing in the number of ethylene glycol (EG) in side chain (PEGMA5 and PEGMA45). The authors found that for PEGMA5, the reactivity ratios for the conventional free-radical copolymerization did not vary with overall monomer conversion (up to $60 \%$ conversion) and the values were close for both monomers, but below $1\left(r_{P E G M A}=0.81 ; r_{M A A}=0.60\right)$. Although slightly different (the ellipsoids at $95 \%$ confidence interval did not overlap), the reactivity ratios in RAFT copolymerization conditions were similar to FRP $\left(r_{P E G M A}=0.59 ; r_{M A A}=0.68\right)$. In contrast, for PEGMA45 the results were substantially different and in FRP the reactivity ratios substantially change with conversion; $r_{M A A}$ decreased (from 1.83 at $10 \%$ to 1.25 at $60 \%$ conversion) and $r_{\text {PEGMA45 }}$ increased (from 0.31 at $10 \%$ to 1.55 at $60 \%$ conversion). For RAFT copolymerization, this effect of the conversion was not found and the values of the reactivities were smaller for both monomers and closer between them.

In this work, the copolymerization of PEGMA macromonomers (with different EG side chain lengths) and MAA in aqueous solution under conditions where MAA is fully ionized is investigated. The principal aim of this study is to understand the copolymerization kinetics of these two monomers that, as discussed above, have shown dependence of the homopropagation rate constant on monomer concentration (MAA and PEGMA), and also of the $\mathrm{pH}$ (MAA) of the aqueous phase. We aim at determining the reactivity ratios of the comonomer system under fully ionized conditions of the MAA and at varying initial monomer concentrations (solids content) for two PEGMAs with increasing number of EG groups in the side chain (PEGMA5 and PEGMA23). The knowledge of the reactivity ratios will help in developing an accurate and predictive mathematical model of the copolymerization process that is needed to develop advanced control strategies for the control of the microstructure of these copolymers.

The article is organized as follows: first, the in situ proton nuclear magnetic resonance spectroscopy ( $\left.{ }^{1} \mathrm{H}-\mathrm{NMR}\right)$ technique used to monitor the copolymerizations of MAA and PEGMA in basic conditions is described. Second, the approach used to estimate the reactivity ratios is briefly described and, finally, the results for the two systems studied (MAA/PEGMA5 and MAA/PEGMA23) are presented and compared in the context of recent literature works for other copolymerization systems presenting similar features.

\section{Materials and Methods}

\subsection{Materials}

Methacrylic Acid (MAA) 99\% with 250 ppm hydroquinone monomethyl ether (MEHQ) as inhibitor and Polyethylene glycol methyl ether methacrylate (PEGMA) (average $\mathrm{Mn} 300 \mathrm{~g} / \mathrm{mol}$ "PEGMA5") with 100 ppm MEHQ and 300 ppm butylated hydroxyl toluene (BHT) as inhibitors were purchased from Sigma-Aldrich (St. Louis, MO, USA) and used as received. Visiomer MPEG 1000 MA W (Methoxy polyethylene glycol 1000 methacrylate 50\% water solution "PEGMA23") with 200 ppm MEHQ as inhibitor was kindly supplied by Evonik Industries (Essen, Germany) Industries and used as received. Potassium persulfate (KPS) $>99 \%$, sodium bicarbonate $\left(\mathrm{NaHCO}_{3}\right)$ and sodium hydroxide $(\mathrm{NaOH})>98 \%$ in pellets were also purchased from Aldrich and used as received. Deuterium oxide $\left(\mathrm{D}_{2} \mathrm{O}\right)>99.9 \%$ was purchased from Euriso-Top (Saint-Aubin Cedex, France). Mili-Q quality water was employed to prepare the solutions.

\subsection{Copolymerization Reactions}

Aqueous-phase (mixture of $\mathrm{H}_{2} \mathrm{O} / \mathrm{D}_{2} \mathrm{O}$ ) free-radical copolymerization reactions of MAA and PEGMA were carried out in NMR tubes and the copolymerization reactions were in situ monitored. 
Comonomer solutions were prepared at different solids contents (5-20 wt \%) with different monomer molar ratios (MAA/PEGMA5: 3/1, 2/1, 1/1 and MAA/PEGMA23: 9/1, 6/1,3/1). Comonomer mixture solutions were neutralized with a $30 \% \mathrm{NaOH}$ solution and kept at a $\mathrm{pH}$ between 7 and 9 , ensuring total ionization of the carboxylic monomer in all cases. $\mathrm{NaHCO}_{3}$ was used as buffer at $1 / 1 \mathrm{~mol}$ ratio with respect to the initiator. All reactions were initiated with a water-soluble thermal initiator (potassium persulfate, KPS) at a concentration of $1 \mathrm{wt} \%$ based on monomer. Higher MAA/PEGMA monomer mol ratios were employed for the longer PEGMA (PEGMA23) because the difference in molecular weight between comonomers will lead to very low initial weight fraction of methacrylic acid $\left(W_{M A A, 0}\right)$ and hence very low rate of polymerization [21]. Note that the monomer molar ratios expressed here are the nominal values; the actual monomer molar ratios were calculated from the vinyl peak areas of each monomer before starting the reaction ( $t_{0}$ in the NMR tube).

The liquid ${ }^{1} \mathrm{H}-\mathrm{NMR}$ spectra were recorded on a Bruker 500 AVANCE $(500 \mathrm{MHz})$ equipped with a $Z$ gradient Broadband observe (BBO) probe. The kinetic study of the reaction was conducted at $343 \mathrm{~K}$ using ${ }^{1} \mathrm{H}$ spectra with suppress of the solvent using WATERGATE sequence. The spectrum was recorded every 2 min during the first $16 \mathrm{~min}$ and then every 10 or $15 \mathrm{~min}$ for $3 \mathrm{~h}$. Monomer conversion of MAA and PEGMA were calculated based on the evolution of the peaks corresponding to the vinyl protons of MAA $(\delta, 5.60,5.25, \mathrm{ppm})$ and PEGMA $(\delta, 6.10,5.70, \mathrm{ppm})$. The peak related to the methoxy group protons $(\delta, 3.30, \mathrm{ppm})$, which is present in the PEGMA macromonomer side chain, was selected as internal reference [36]. Figure 1 illustrates the change on the intensity of the MAA and PEGMA peaks over copolymerization time. The spectra were processed by the software MestRe Nova 9.0 (MestreLab Research Chemistry Software solutions, Santiago de Compostela, Spain).

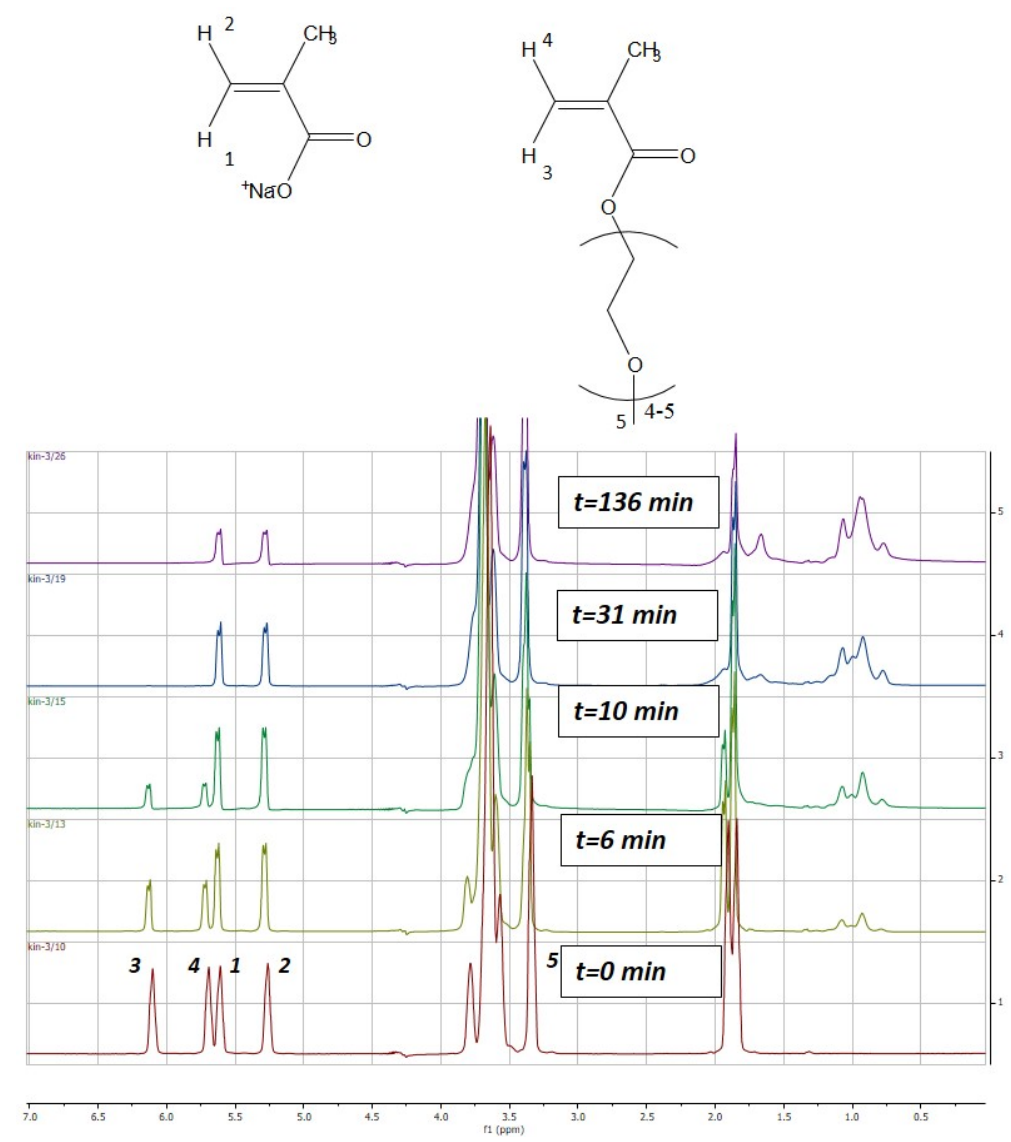

Figure 1. Time evolution of the proton nuclear magnetic resonance $\left({ }^{1} \mathrm{H}-\mathrm{NMR}\right)$ spectra of the aqueous-solution copolymerization of MAA and PEGMA5 carried out at $70{ }^{\circ} \mathrm{C}(S C=10 \mathrm{wt} \%$ MAA/PEGMA5 = 1/1). MAA = methacrylic acid; PEGMA = poly(ethylene glycol) methyl ether methacrylate; $S C=$ solids content. 


\subsection{Estimation of Reactivity Ratios}

Free-radical copolymerization leads to the formation of copolymers in which the distribution of the monomers in the chain is governed by kinetics. There is considerable experimental evidence [37-39] showing that, in many copolymerization systems, propagation depends on the nature of the monomer and on the last two units of the growing chain. This is referred to as penultimate model. Nevertheless, copolymer composition can be well described by considering a model in which the reactivity of the propagation reaction is governed by the nature of the monomer and the terminal unit of the polymer radical (terminal model). In this work, the later assumption was considered to describe the copolymerization of MAA and PEGMA macromonomers. The reactivity ratios were estimated using the evolution of individual comonomer conversions over overall conversion following the method developed by De la Cal et al. [40,41]. This method is briefly described below:

Methacrylic acid (MAA) and polyethylene glycol methacrylate (PEGMA) will be represented with letters $A$ and $B$, respectively. The material balances for each of the monomers in a batch reactor, considering that terminal model kinetics is applied, can be written as:

$$
\begin{aligned}
& \frac{d[A]}{d t}=-R_{p A}=-\left(k_{p A A} P_{A}+k_{p B A} P_{B}\right)[A]\left[R^{*}\right] \\
& \frac{d[B]}{d t}=-R_{p B}=-\left(k_{p A B} P_{A}+k_{p B B} P_{B}\right)[B]\left[R^{*}\right]
\end{aligned}
$$

In Equations (1) and (2) [i] is concentration of monomer $i(\mathrm{~mol} / \mathrm{L}), R_{p i}$, the polymerization rate of monomer $i(\mathrm{~mol} / \mathrm{L} \cdot \mathrm{s}), k_{p i j}$, the propagation rate constant of radicals of terminal unit $i$ with monomer $j(\mathrm{~L} / \mathrm{mol} \cdot \mathrm{s}), P_{i}$ the probability of finding active chain with ultimate unit of type $i$, and $R^{*}$ is the total concentration of radicals (mol/L).

Considering the Quasi-Steady-State assumption (QSSA) is fulfilled, the probabilities are defined as follows:

$$
\begin{gathered}
P_{A}=\frac{k_{p B A}[A]}{k_{p B A}[A]+k_{p A B}[B]} \\
P_{B}=1-P_{A}
\end{gathered}
$$

MAA conversion and overall conversion are defined as:

$$
\begin{gathered}
X_{A}=\frac{[A]_{0}-[A]}{[A]_{0}} \\
X_{T}=\frac{\left([A]_{0}-[A]\right)+\left([B]_{0}-[B]\right)}{[A]_{0}+[B]_{0}}
\end{gathered}
$$

where $[A]_{0}$ and $[B]_{0}$ are initial concentration of MAA and PEGMA, respectively. Thus:

$$
\begin{gathered}
d X_{A}=-\frac{d[A]}{[A]_{0}} \\
d X_{T}=\frac{-d[A]-d[B]}{[A]_{0}+[B]_{0}} \\
\frac{d X_{A}}{d X_{T}}=\frac{[A]_{0}+[B]_{0}}{[A]_{0}} \frac{R_{p A}}{R_{p A}+R_{p B}}=\frac{[A]_{0}+[B]_{0}}{[A]_{0}}\left(\frac{1+r_{A} \frac{[A]}{[B]}}{2+r_{A} \frac{[A]}{[B]}+r_{B} \frac{[B]}{[A]}}\right)
\end{gathered}
$$

where $r_{A}$ and $r_{B}$ are the reactivities of MAA and PEGMA defined as:

$$
r_{A}=\frac{k_{p A A}}{k_{p A B}}
$$




$$
r_{B}=\frac{k_{p B B}}{k_{p B A}}
$$

To integrate Equation (9), concentrations can be expressed as a function of $X_{A}$ and $X_{T}$ employing Equations (5) and (6):

$$
\frac{[A]}{[B]}=\frac{[A]_{0}\left(1-X_{A}\right)}{[B]_{0}-X_{T}\left([A]_{0}+[B]_{0}\right)+[A]_{0} X_{A}}
$$

The cumulative composition can be determined as a function of the individual conversion of MAA, $X_{A}$, and the overall conversion as follows:

$$
Y_{A}=\frac{[A]_{0} X_{A}}{\left([A]_{0}+[B]_{0}\right) X_{T}}
$$

The reactivity ratios $r_{A}$ and $r_{B}$ can be estimated using a parameter estimation algorithm that minimizes the objective function of Equation (14), where $Y_{\text {Aexp }}$ is the experimentally measured cumulative composition referred to MAA determined by in situ ${ }^{1} \mathrm{H}-\mathrm{NMR}$, and $Y_{\text {Acal }}$ is the theoretically determined cumulative composition calculated using the set of Equations (9)-(13) and the initial concentrations of the comonomers. The subscript $i$ makes reference to the experiment, and subscript $j$ to the sample number of each of the experiments used in the estimation procedure. The only parameters of the model are the reactivity ratios.

$$
J=\left[\sum_{i=1}^{N} \sum_{j=1}^{P i}\left(Y_{\text {Aexp }}-Y_{\text {Acal }}\right)^{2}\right]
$$

Parameter estimation was carried out using a direct-search estimation algorithm employing subroutine DBCPOL and the subroutine for solving ordinary differential equations DIVPRK from IMSL library.

\section{Results and Discussion}

The aqueous-phase solution copolymerization of two comonomer systems was studied: MAA-co-PEGMA5 and MAA-co-PEGMA23; namely, polyethylene glycol methyl methacrylate monomers with 5 and 23 ethylene glycol (EG) units.

\subsection{MAA-co-PEGMA5 (5 Ethylene Glycol Units)}

Copolymerizations of MAA and PEGMA5 were carried out in NMR tubes at different comonomer ratios (MAA/PEGMA: 3/1, 2/1 and 1/1) and different solids content (SC: 5, 10, 15 and $20 \mathrm{wt} \%$ ).

Figure 2 shows that conversions of both comonomers increase with solids content (for MAA, the effect was more clear at higher MAA/PEGMA ratios), and that PEGMA5 is more reactive than MAA at fully ionized conditions. The effect of the solids content on the conversion indicates that the dependence of the polymerization rate on the monomer conversion is not first order for any of the two monomers. This has been reported for the aqueous solution homopolymerization of MAA [19-21,25,42]. It has been found that the propagation rate coefficient of MAA was a function of the concentration of the monomer in the aqueous phase and of the ionization degree. Under fully ionized conditions (like in this work) the propagation rate coefficient of MAA increases with monomer weight fraction, but under non-ionized and partially ionized the propagation rate constant decreases with increasing monomer fraction.

Recently, Smolne and co-workers have studied the propagation and termination kinetics of PEG-lated methacrylates in aqueous solution [1]. Similar to other water-soluble monomers, it has been also found that the kinetics of the polymerization is affected by the monomer weight fraction in the aqueous solution. The propagation rate coefficient increased as the solids content decreased, which follows the same trend as the polymerization of non-ionized or partially ionized MAA. In the copolymerizations of Figure 2, the trends of the overall propagation rate constants for each monomer are like for the fully ionized MAA; namely, the overall propagation rate increases with solids content. 

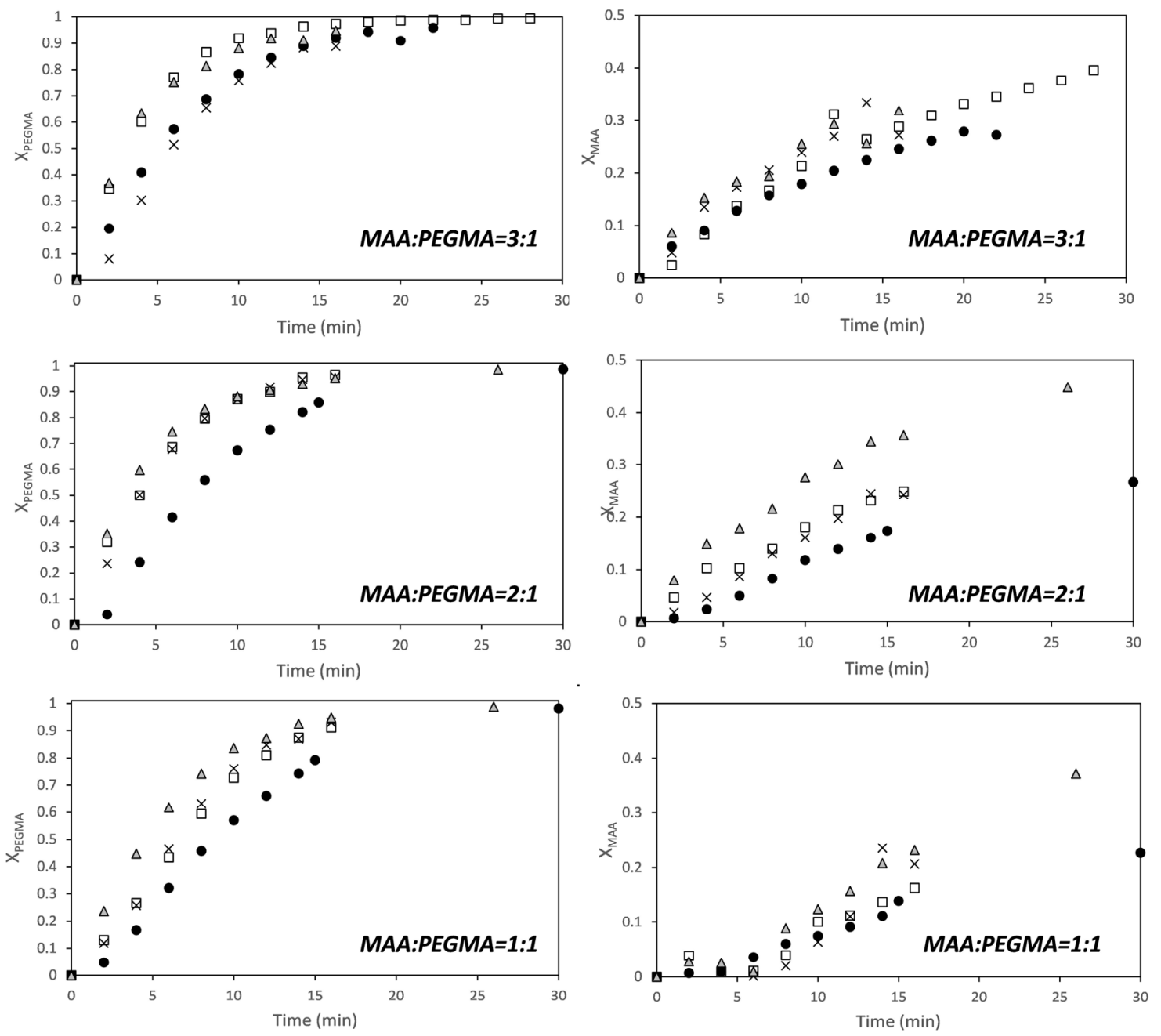

Figure 2. Time evolution of the conversion of MAA (right) and PEGMA 5 (left) for different monomer ratios (MAA/PEGMA as indicated in the figures) at different solids content (SC): $\bullet-5 \mathrm{wt} \%$; $\square-10 \mathrm{wt} \%$; $\times-15$ wt $\%$; $\Delta-20$ wt $\%$.

The faster conversion of the PEGMA5 macromonomer can be attributed to a substantial difference in reactivity ratios. As discussed in the introduction, there are no reports for the reactivity ratios of this comonomer system calculated in aqueous phase at fully ionized conditions of the MAA monomer. However, reactivity ratios of AA and AM in aqueous phase at fully ionized conditions have been investigated by several groups [8,43-46]. Preusser et al. have done an extensive experimental analysis including copolymerization in a broad range of initial monomer concentrations and degrees of ionization and have determined the reactivity ratios. They found that the reactivity ratio of each monomer is a function of initial weight fraction of monomer and of the ionization degree and they provided an empirical equation that captures well the combined effect of both variables [8]. The reactivity ratios for the MAA/PEGMA5 comonomer system were estimated from these data using the algorithm presented above. Figure 3 shows the comparison between the cumulative compositions of MAA determined experimentally from the NMR data (dots) and the estimated one (lines) for the estimated reactivity ratios at each solids content. Note that the reactivity ratios were initially estimated at each solids content because of the effects observed on the individual monomer conversions shown in Figure 2, and also because a similar effect was found for the AA and AM comonomer system [8]. 

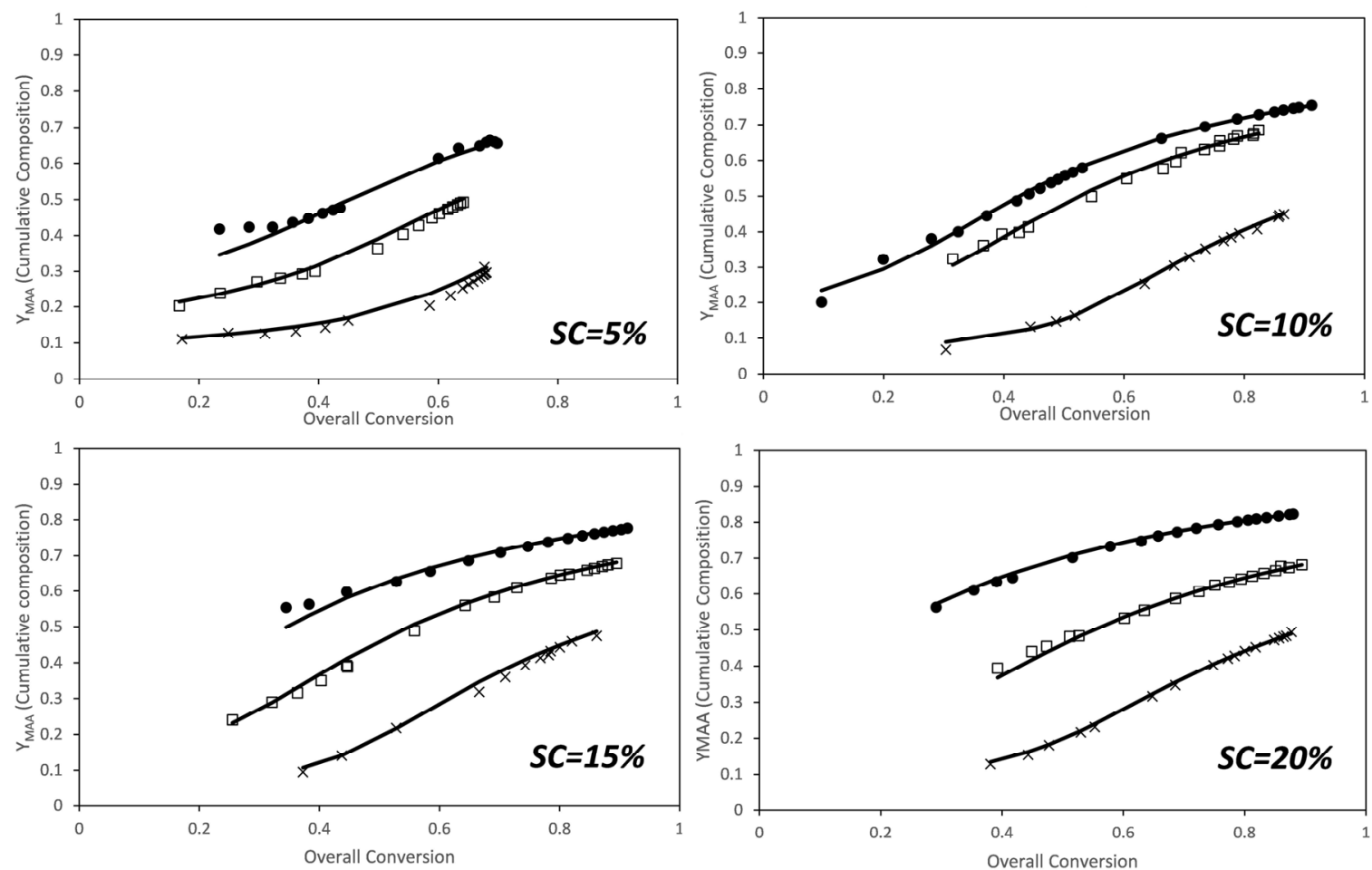

Figure 3. Conversion evolution of the cumulative composition of MAA for the in situ nuclear magnetic resonance (NMR) experiments. (Dots) experimental results, (lines) model predictions for the estimated reactivity ratios at each solids content (see Figure 4 ). Molar ratios: $x-1 / 1 ; \square-2 / 1 ; \bullet-3 / 1$.

Figure 4 shows the estimated reactivity ratios at each solids content. It can be seen that the reactivity ratio of each comonomer increases with solids content (in the range studied in this work; note that the solids content analysed is limited by the viscosities produced during this polymerization carried out at $70{ }^{\circ} \mathrm{C}$ ). This unexpected dependence of the reactivity with solids content has been recently found for the aqueous-phase copolymerization of AA and AM [8], a comonomer system that shared similar features on the dependence of the propagation rate coefficients with initial monomer concentration and ionization degree as the monomers investigated in this work.

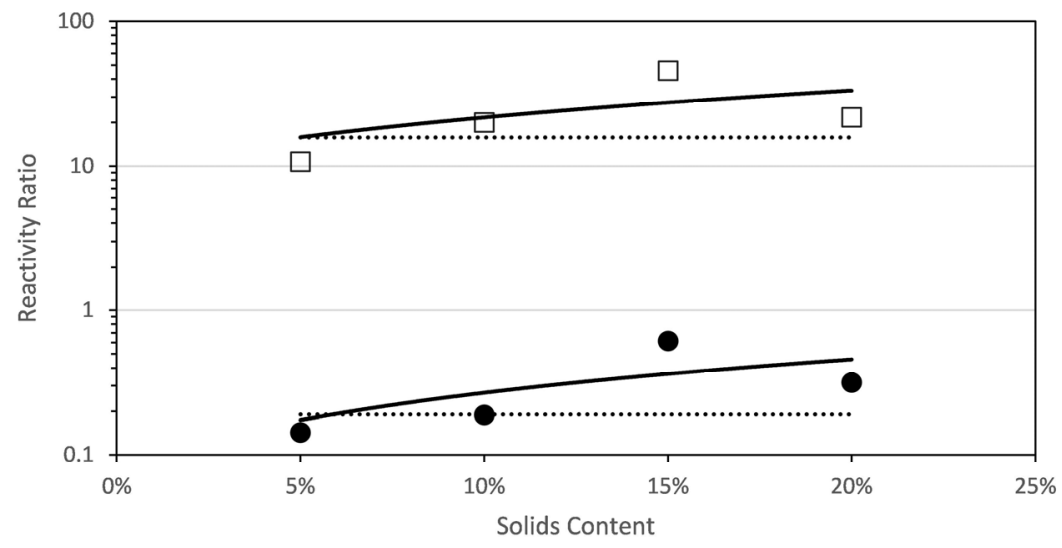

Figure 4. Reactivity ratios for the copolymerization of fully ionized MAA and PEGMA5 as determined by a global fit to the combined data (dashed line) and by fitting data at each initial solids content (squares and circles). A linear fit of the reactivity ratios estimated at each solids content is included (solid line) $\square r_{P E G M A} ; \bullet r_{M A A}$. 
Figure 4 plots the reactivity ratios estimated at each initial solids content and, for comparison purpose, the reactivity ratio estimated to fit globally all the experimental data gathered at different initial concentrations and monomer ratio is included. It was found that fitting the experimental data with just a pair of reactivity ratios (global fitting) was not appropriate, because the value of the objective function was almost one order of magnitude higher as compared with the objective functions of any of the individual fittings. The reactivity ratios estimated at each solids content were fitted with a linear equation that is also drawn in Figure 4 and detailed in the following equations. Note that the regressions of the linear fits are not excellent and hence the equations below should be considered only as a reasonable approximation.

$$
\begin{aligned}
r_{M A A} & =0.07+1.89 \cdot S C_{0} \\
r_{P E G M A} & =9.89+117.21 \cdot S C_{0}
\end{aligned}
$$

where $S C_{0}$ is the initial weight fraction of comonomers in the copolymerization formulation $\left(0<S C_{0}<1\right)$.

\subsection{MAA-co-PEGMA23 (23 Ethylene Glycol Units)}

In this part of the work, we aimed to assess the effect of a longer lateral chain of the PEGMA macromonomer on the reactivity ratios with MAA under fully ionized conditions.

As in the previous set of reactions, the polymerizations were carried out under fully ionized conditions of the MAA; however, for this pair of monomers lower solids content were employed $(5,7.5$ and $10 \mathrm{wt} \%$ ) to avoid excessively high viscosities in the NMR tube and hence inhomogeneity in the reaction medium and unreliable results.

According to Krivorotova et al. [35], in a mixture of $\mathrm{D}_{2} \mathrm{O} /$ Dioxane and with non-ionized MAA increasing the size of the lateral chain of the PEGMA (from PEGMA5 to PEGMA45), substantial differences were found on the reactivity ratios of this comonomer pair. Whereas the reactivities were found independent of conversion for the short PEGMA, for the long PEGMA the reactivity of MAA (that was higher than that of PEGMA45) decreased and that of PEGMA45 increased making them to be close at higher conversion.

Figure 5 shows the time evolution of the individual conversions of MAA and PEGMA23 monitored by in situ ${ }^{1} \mathrm{H}-\mathrm{NMR}$. The trends are very similar to those observed for PEGMA5; namely, PEGMA23 macromonomer is more reactive than the MAA at fully ionized conditions, and the dependence of the polymerization rate on the solids content is not of first order for any of the two monomers. For the sake of comparison, the reactivity ratios of this pair has been estimated using the approach described above at each solids content and also using the whole set of data.

Figure 6 shows the comparison between the cumulative compositions of MAA experimentally determined from the NMR (dots) and the estimated cumulative compositions (lines) calculated for the estimated reactivity ratios at each solids content. Fitting of the cumulative composition for the set of experiments carried out at different solids content and monomer ratios is excellent.

The estimated reactivity ratios at different solids content for the combined data are shown in Figure 7. There are noticeable differences with respect to the short PEGMA. First, none of the reactivity ratios increase with solids content; on the contrary, $r_{P E G M A}$ decreases and $r_{M A A}$ is very similar for all the solids content. Indeed, the single pair of the reactivity ratios obtained by globally fitting all the data (the dashed lines) is very close to the values obtained in the individual fitting except for the values at $10 \%$ solids content. The linear fitting of the $r_{P E G M A}$ and $r_{M A A}$ calculated at each solids content provide the following empirical equations for dependence of the reactivity ratios with the solids content.

$$
\begin{gathered}
r_{M A A}=0.14-0.14 \cdot S C_{0} \\
r_{P E G M A}=26.56-153.12 \cdot S C_{0}
\end{gathered}
$$



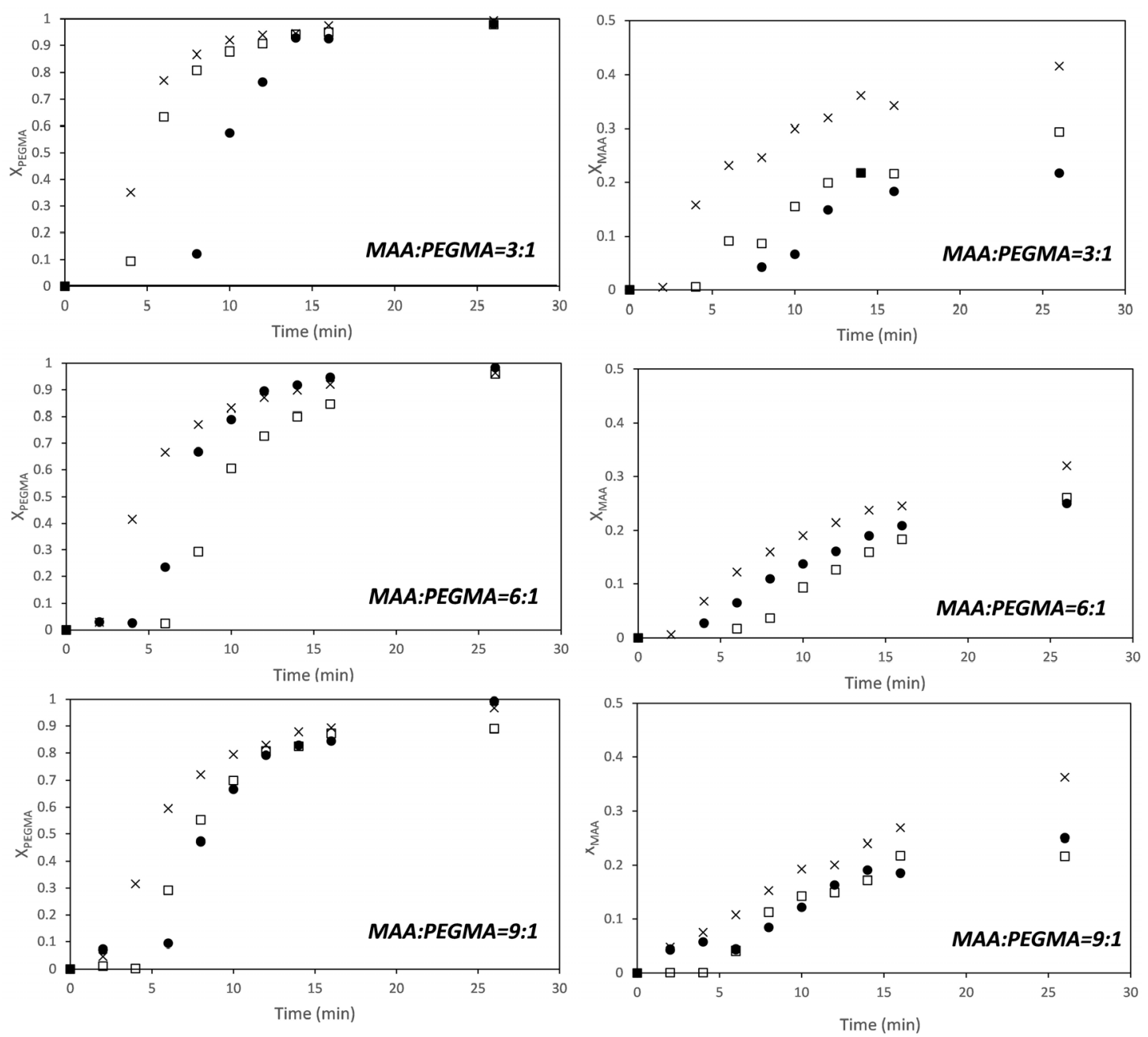

Figure 5. Time evolution of the conversion of MAA (right) and PEGMA 23 (left) at monomer ratios (MAA/PEGMA23 as indicated in the figure) at different solids content. $\bullet-5 \mathrm{wt} \%$; $\square-7.5 \mathrm{wt} \%$; $\times-10$ wt $\%$.

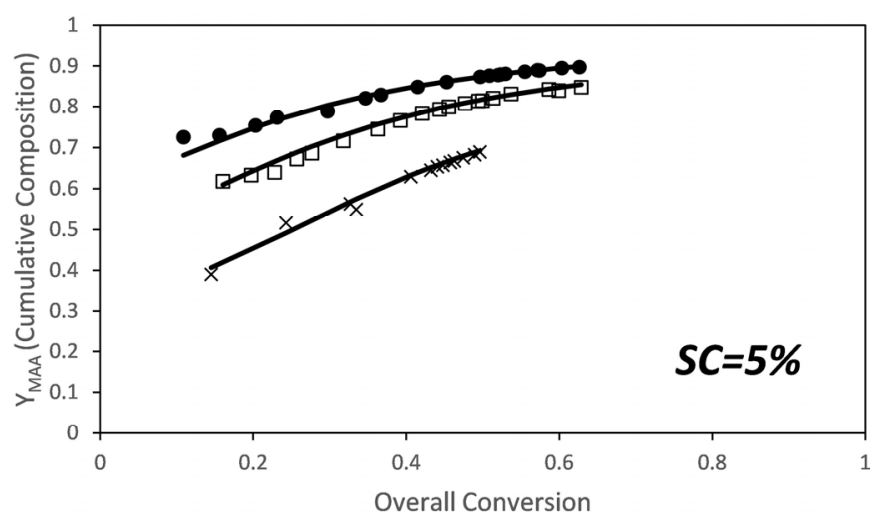

Figure 6. Cont. 

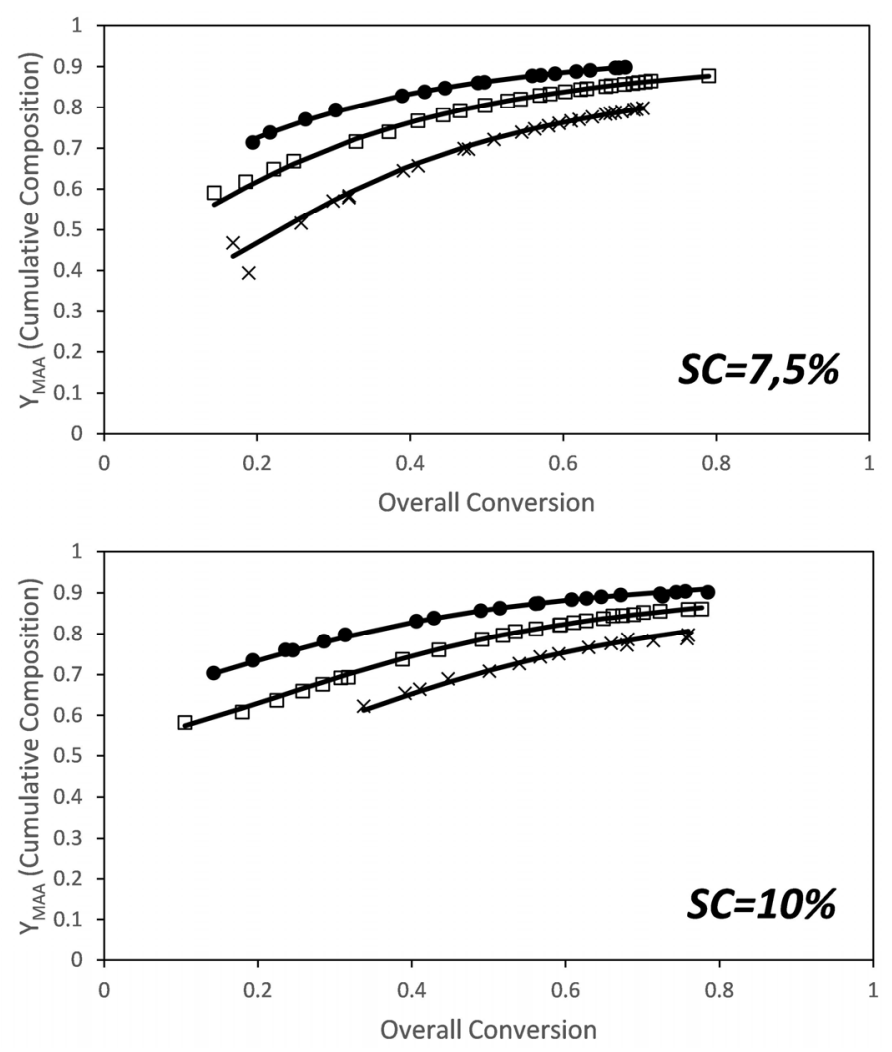

Figure 6. Conversion evolution of the cumulative composition of MAA for the in situ NMR experiments. (Dots) experimental results, (lines) model predictions for the estimated reactivity ratios. Molar ratios: $\times-3 / 1 ; \square-6 / 1 ; \bullet-9 / 1$.

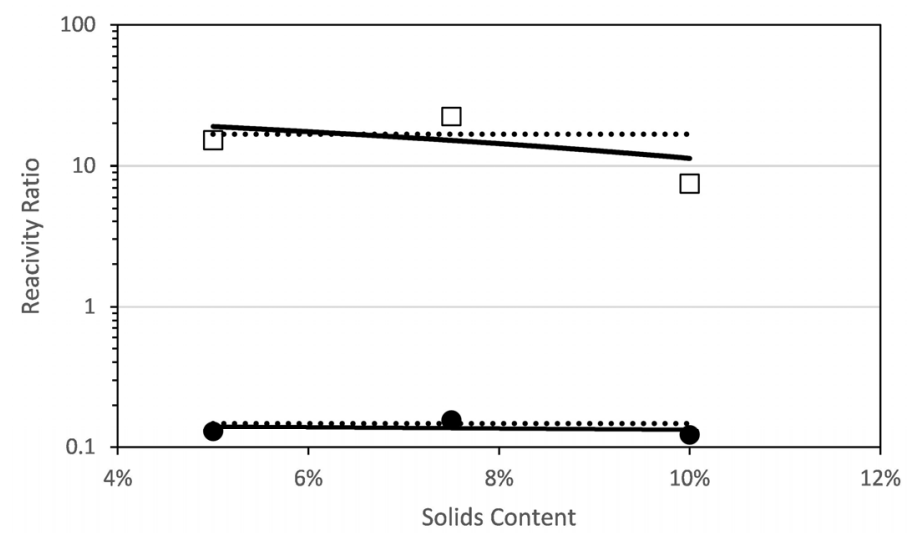

Figure 7. Reactivity ratios for the copolymerization of fully ionized MAA and PEGMA23 as determined by a global fit to the combined data (dashed line) and by fitting data at each initial solids content (squares and circles). A linear fit of the reactivity ratios at each solids content is included (solid line)

$\square r_{P E G M A} ; \bullet r_{M A A}$.

\section{Conclusions}

The aqueous-phase copolymerization kinetics of MAA and PEGMA macromonomers (with two lateral chains of different number of EG groups, PEGMA5 and PEGMA23) under fully ionized conditions of MAA at different initial monomer ratios and initial overall monomer concentrations (solids content) were monitored by in situ NMR. The rate of consumption of both monomers indicates that PEGMA macromonomer is more reactive than MAA, and that the rates of consumption of both 
monomers are not first order with respect to the concentration of the monomer in agreement with recent results that demonstrated that, like for other water-soluble monomers, the propagation rate coefficient of these monomers depends on the concentration of the monomer.

The reactivity ratios estimated from the copolymerization kinetics revealed that this dependency of the propagation rate coefficient on the monomer concentration was also translated to the reactivity ratios of these monomers. Thus, it was found that for the short PEGMA the reactivity ratios $r_{M A A}$ and $r_{P E G M A}$ increased with the solids content. Interestingly, this trend was not maintained for the longer PEGMA, whose reactivity ( $\left.r_{P E G M A 23}\right)$ decreased with conversion, whereas the reactivity ratio of MAA remained roughly constant with the longer PEGMA.

For both systems, empirical linear fits of the reactivity ratios with the solids content were calculated that can be easily implemented in modelling and control schemes aimed at controlling the microstructure of the comb-like copolymers.

Acknowledgments: This work has been carried out in the framework of the BASKRETE initiative under the umbrella of the EUSKAMPUS project. Iñaki Emaldi acknowledges the funding provided by EUSKAMPUS Fundazioa, POLYMAT and TECANLIA for his scholarship. Shaghayegh Hamzehlou and Jose Ramon Leiza acknowledge the funding provided by MINECO (CTQ 2014-59016P) and Basque Government (IT-999-16). Jorge Sánchez Dolado acknowledges the funding for the GEI Green Concrete Project given by the Basque Government (2015 Emaitek Program). The authors also thank the discussion with José Carlos de la Cal on the estimation of the reactivity ratios and they are grateful to José Ignacio Miranda and the SGIker Gipuzkoa Unit (UPV /EHU) for the NMR facilities.

Author Contributions: I.E. and S.H. performed the experiments. I.E., J.R.L. and J.S.-D. conceived the experiments. I.E., S.H. and J.R.L. wrote the paper.

Conflicts of Interest: The authors declare no conflict of interest.

\section{References}

1. Smolne, S.; Weber, S.; Buback, M. Propagation and termination kinetics of poly(ethylene glycol) methyl ether methacrylate in aqueous solution. Macromol. Chem. Phys. 2016, 217, 2391-2401. [CrossRef]

2. Neugebauer, D. Graft copolymers with poly(ethylene oxide) segments. Polym. Int. 2007, 56, 1469-1498. [CrossRef]

3. Robinson, D.N.; Peppas, N.A. Preparation and characterization of pH-responsive poly (methacrylic acid-g-ethylene glycol) nanospheres. Macromolecules 2002, 35, 3668-3674. [CrossRef]

4. Tuncel, A. Emulsion copolymerization of styrene and poly(ethylene glycol) ethyl ether methacrylate. Polymer 2000, 41, 1257-1267. [CrossRef]

5. Halake, K.; Birajdar, M.; Kim, B.S.; Bae, H.; Lee, C.; Kim, Y.J.; Kim, S.; Kim, H.J.; Ahn, S.; An, S.Y.; et al. Recent application developments of water-soluble synthetic polymers. J. Ind. Eng. Chem. 2014, 20, 3913-3918. [CrossRef]

6. Brown, R.; Stitzel, B.; Sauer, T. Steric stabilization by grafting and copolymerization of water soluble oligomers and polymers. Macromol. Chem. Phys. 1995, 196, 2047-2064. [CrossRef]

7. Colombo, C.; Gatti, S.; Ferrari, R.; Casalini, T.; Cuccato, D.; Morosi, L.; Zucchetti, M.; Moscatelli, D. Self-assembling amphiphilic PEGylated block copolymers obtained through RAFT polymerization for drug-delivery applications. J. Appl. Polym. Sci. 2016, 133, 1-8. [CrossRef]

8. Preusser, C.; Ezenwajiaku, I.H.; Hutchinson, R.A. The combined influence of monomer concentration and ionization on acrylamide/acrylic acid composition in aqueous solution radical batch copolymerization. Macromolecules 2016, 49, 4746-4756. [CrossRef]

9. Amjad, Z. Water Soluble Polymers. Solution properties and applications; Kluwer Academic Press: New York, NY, USA, 2002.

10. Dautzenberg, H.; Jaeger, W.; Kötz, J.; Phillip, B.; Seidel, C.; Stscherbina, D. Polyelectrolytes: Formation, Characterisation and Application; Hauser Publishers: Munich, Germany, 1994.

11. Yu., K.E. Water Soluble Poly-N-Vinylamides Synthesis and Physiochemical Properties; John Wiley and Sons Ltd.: Chichester, UK, 1998.

12. Kricheldorf, H.R. Handbook of Polymer Synthesis; Mercel Dekker Inc.: New York, NY, USA, 1991. 
13. Lacik, I.; Beuermann, S.; Buback, M. PLP-SEC study into the free-radical propagation rate coefficients of partially and fully ionized acrylic acid in aqueous solution. Macromol. Chem. Phys. 2004, 205, 1080-1087. [CrossRef]

14. Lacík, I.; Beuermann, S.; Buback, M. Aqueous phase size-exclusion-chromatography used for PLP-SEC studies into free-radical propagation rate of acrylic acid in aqueous solution. Macromolecules 2001, 34, 6224-6228. [CrossRef]

15. Lacík, I.; Beuermann, S.; Buback, M. PLP-SEC study into free-radical propagation rate of nonionized acrylic acid in aqueous solution. Macromolecules 2003, 36, 9355-9363. [CrossRef]

16. Cutie, S.S.; Smith, P.B.; Henton, D.E.; Staples, T.L.; Powell, C. Acrylic acid polymerization kinetics. J. Polym. Sci. Part A Polym. Chem. 1997, 35, 2029-2047. [CrossRef]

17. Seabrook, S.A.; Tonge, M.P.; Gilbert, R.G. Pulsed laser polymerization study of the propagation kinetics of acrylamide in water. J. Polym. Sci. Part A Polym. Chem. 2005, 43, 1357-1368. [CrossRef]

18. Lacík, I.; Chovancova, A.; Uhelsk, L.; Preusser, C.; Hutchinson, R.A.; Buback, M. PLP-SEC studies into the propagation rate coefficient of acrylamide radical polymerization in aqueous solution. Macromolecules 2016, 49, 3244-3253. [CrossRef]

19. Beuermann, S.; Buback, M.; Hesse, P.; Lacík, I. Free-radical propagation rate coefficient of nonionized methacrylic acid in aqueous solution from low monomer concentrations to bulk polymerization. Macromolecules 2006, 39, 184-193. [CrossRef]

20. Beuermann, S.; Buback, M.; Hesse, P.; Kukućková, S.; Lacík, I. Propagation rate coefficient of non-ionized methacrylic acid radical polymerization in aqueous solution. The effect of monomer conversion. Macromol. Symp. 2007, 248, 41-49. [CrossRef]

21. Lacík, I.; Učňová, L.; Kukučková, S.; Buback, M.; Hesse, P.; Beuermann, S. Propagation rate coefficient of free-radical polymerization of partially and fully ionized methacrylic acid in aqueous solution. Macromolecules 2009, 42, 7753-7761. [CrossRef]

22. Stach, M.; Lacík, I.; Chorvát, D.; Buback, M.; Hesse, P.; Hutchinson, R.A.; Tang, L. Propagation rate coefficient for radical polymerization of $\mathrm{N}$-vinyl pyrrolidone in aqueous solution obtained by PLP-SEC. Macromolecules 2008, 41, 5174-5185. [CrossRef]

23. Stach, M.; Lacík, I.; Kasák, P.; Chorvát, D.; Saunders, A.J.; Santanakrishnan, S.; Hutchinson, R.A. Free-radical propagation kinetics of $\mathrm{N}$-vinyl formamide in aqueous solution studied by PLP-SEC. Macromol. Chem. Phys. 2010, 211, 580-593. [CrossRef]

24. Santanakrishnan, S.; Hutchinson, R.A.; Učňová, L.; Stach, M.; Lacík, I.; Buback, M. Polymerization kinetics of water-soluble $N$-vinyl monomers in aqueous and organic solution. Macromol. Symp. 2011, 302, $216-223$. [CrossRef]

25. Buback, M.; Hesse, P.; Hutchinson, R.A.; Lacík, I.; Kasák, P.; Stach, M.; Utz, I. Kinetics and modeling of free-radical batch poymerization of nonionized methacrylic acid in aqueous solution. Ind. Eng. Chem. 2008, 47, 8197-8204. [CrossRef]

26. Flatt, R.; Schober, I. Superplasticizers and the Rheology of Concrete 7. Understanding the Rheology of Concrete; Roussel, N., Ed.; Woodhead Publishing Limited: Oxford, UK, 2011.

27. Marchon, D.; Sulser, U.; Eberhardt, A.; Flatt, R.J. Molecular design of comb-shaped polycarboxylate dispersants for environmentally friendly concrete. Soft Matter 2013, 9, 10719-10728. [CrossRef]

28. Guicquero, J.P.; Maitrasse, P.; Mosquet, M.A.; Alphonse, S. A Water Soluble or Water Dispersible Dispersing Agent. FR2776285, 19 March 1998.

29. Lei, L.; Plank, J. Synthesis and properties of a vinyl ether-based polycarboxylate superplasticizer for concrete possessing clay tolerance. Ind. Eng. Chem. Res. 2014, 53, 1048-1055. [CrossRef]

30. Plank, J.; Pöllmann, K.; Zouaoui, N.; Andres, P.R.; Schaefer, C. Synthesis and performance of methacrylic ester based polycarboxylate superplasticizers possessing hydroxy terminated poly(ethylene glycol) side chains. Cem. Concr. Res. 2008, 38, 1210-1216. [CrossRef]

31. Plank, J.; Sakai, E.; Miao, C.W.; Yu, C.; Hong, J.X. Chemical admixtures-Chemistry, applications and their impact on concrete microstructure and durability. Cem. Concr. Res. 2015, 78, 81-99. [CrossRef]

32. Pourchet, S.; Liautaud, S.; Rinaldi, D.; Pochard, I. Effect of the repartition of the PEG side chains on the adsorption and dispersion behaviors of PCP in presence of sulfate. Cem. Concr. Res. 2012, 42, 431-439. [CrossRef] 
33. Smith, B.L.; Klier, J. Determination of monomer reactivity ratios for copolymerizations of methacrylic acid with poly(ethylene glycol) monomethacrylate. J. Appl. Polym. Sci. 1998, 68, 1019-1025. [CrossRef]

34. Georges, S.; Hamaide, T. Determination of the reactivity ratios of the methacrylic acid-polyethylene glycol methacrylate system by aqueous SEC. Bul. Stiint. al Univ. "Politehnica" din Timisoara 2001, 46, 187-191.

35. Krivorotova, T.; Vareikis, A.; Gromadzki, D.; Netopilík, M.; Makuška, R. Conventional free-radical and RAFT copolymerization of poly(ethylene oxide) containing macromonomers. Eur. Polym. J. 2010, 46, 546-556. [CrossRef]

36. Najafi, V.; Ziaee, F.; Kabiri, K.; Mehr, M.J.Z.; Abdollahi, H.; Nezhad, P.M.; Jalilian, S.M.; Nouri, A. Aqueous free-radical polymerization of PEGMEMA macromer: Kinetic studies via an on-line ${ }^{1} \mathrm{H}$ NMR technique. Iran. Polym. J. Engl. Ed. 2012, 21, 683-688. [CrossRef]

37. Fukuda, T.; Inagaki, H. Free-Radical Copolymerization. 3. Determination of rate constants of propagation and termination for the styrene/methyl methacrylate system. A critical test of terminal-model kinetics 1. Macromolecules 1985, 18, 17-26. [CrossRef]

38. Coote, M.L.; Davis, T.P. Mechanism of the propagation step in free-radical copolymerisation. Prog. Polym. Sci. 1999, 24, 1217-1251. [CrossRef]

39. Olaj, O.F.; Schnöll-Bitai, I.; Kremminger, P. Evaluation of individual rate constants from the chain-length distribution of polymer samples prepared by intermittent (rotating sector) photopolymerization-2. The copolymerization system styrene-methyl methacrylate. Eur. Polym. J. 1989, 25, 535-541. [CrossRef]

40. De La Cal, J.C.; Leiza, J.R.; Asúa, J.M. Estimation of reactivity ratios using emulsion copolymerization data. J. Polym. Sci. Part A Polym. Chem. 1991, 29, 155-167. [CrossRef]

41. García, G. Síntesis de Floculantes Catiónicos en Reactores Continuos. Ph.D. Thesis, University of the Basque Country (UPV/EHU), Donostia-San Sebastián, Spain, 2009.

42. Beuermann, S.; Buback, M.; Hesse, P.; Hutchinson, R.A.; Kukučková, S.; Lacík, I. Termination kinetics of the free-radical polymerization of nonionized methacrylic acid in aqueous solution. Macromolecules 2008, 41, 3513-3520. [CrossRef]

43. Riahinezhad, M.; Mcmanus, N.; Penlidis, A. Effect of monomer concentration and $\mathrm{pH}$ on reaction kinetics and copolymer microstructure of Acrylamide/Acrylic acid copolymer. Macromol. React. Eng. 2015, 9, 100-113. [CrossRef]

44. Paril, A.; Alb, A.M.; Giz, A.T.; Çatagil-Giz, H. Effect of medium pH on the reactivity ratios in acrylamide acrylic acid copolymerization. J. Appl. Polym. Sci. 2007, 103, 968-974. [CrossRef]

45. Ponratnam, S.; Kapur, S.L. Reactivity ratios of ionizing monomers in aqueous solution. Copolymerization of acrylic and methacrylic acids with acrylamide. Makromol. Chem. 1977, 178, 1029-1038. [CrossRef]

46. Cabaness, W.R.; Lin, T.Y.-C.; Párkányi, C. Effect on the $\mathrm{pH}$ on the reactivity ratios in the copolymerization of acrylic acid and acrylamide. J. Polym. Sci. Part A Polym. Chem. 1971, 9, 2155-2170. [CrossRef] 